\section{Blocking without obstruction}

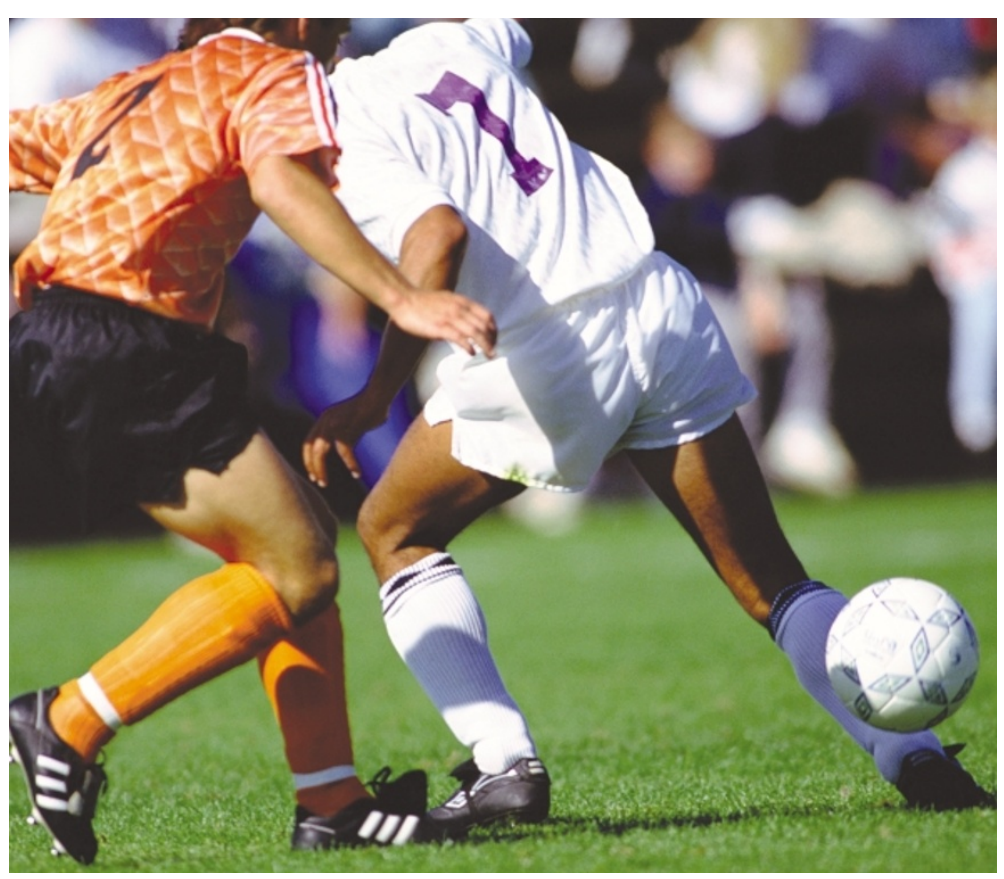

Research in the May issue of Nature Structural Biology has shown that a small molecule known as THC antagonizes oestrogen receptor- $\beta$ (ER- $\beta$ ) by means of a novel mechanism. THC blocks transcription without the usual physical obstruction of the positioning of helix 12 in the ER- $\beta$ protein, which is necessary for receptor activation. Interestingly, whereas THC acts as an antagonist of ER- $\beta$, it is an agonist of ER- $\alpha$. This study solved the crystal structures of THC bound to the ligand-binding domains of both ER- $\alpha$ and ER- $\beta$, in order to investigate the mechanisms of THC agonism and antagonism.

Both ER- $\alpha$ and ER- $\beta$ mediate the physiological effects of both endogenous and synthetic oestrogens. These receptors are members of the nuclear-receptor superfamily of ligand-regulated transcription factors. The ligand-binding domains of ER- $\alpha$ and ER- $\beta$ each have a transcriptional activation function, which is responsive to agonists, including oestrogen $17 \beta$-oestradiol (E2) and diethylstilbestrol. Antagonists, such as 4-hydroxytamoxifen and raloxifene, block the activation-function activity. Agonists stabilize a conformation of the receptor in which helix 12 lies across the opening of the binding pocket, thereby allowing the receptor to interact with the transcriptional coactivators that mediate ligand-dependent transcription of the receptor. Most ER antagonists have bulky side chains that cannot fit within the binding pocket, and so ER- $\beta$ helix 12 cannot adopt the agonist-bound conformation, which occludes binding to transcriptional co-activators. But because THC lacks the bulky side chain that is typical of other ER antagonists, it must antagonize ER- $\beta$ through a different mechanism.

Comparing the crystal structures of THC bound to either ER- $\beta$ or ER- $\alpha$ shows that although THC binds to both chains in a similar manner overall, it fails to stabilize several of the binding-pocket interactions with ER- $\beta$ in comparison to those with ER- $\alpha$. The slight differences in sequence between the two ERs means that crucial residues in ER- $\beta$ adopt a random-coil conformation instead of a helical one. This does not favour the agonist-bound conformation of helix 12 , and actually stabilizes an

\title{
Birds of a feather...
}

Many hits from high-throughput and virtual screening programmes are subsequently found to have peculiar, undesirable characteristics - they act non-competitively, show little relationship between structure and activity, and have poor selectivity. Such hits can waste much time and effort, but despite their common occurrence, the underlying reasons for their behaviour have remained unknown. Now, writing in the Journal of Medicinal Chemistry, McGovern et al. provide evidence that these compounds, although structurally unrelated, share the ability to aggregate, which results in them being falsely detected as hits in screening assays.

Initially, the authors investigated 15 diverse compounds described as inhibitors of one or more protein or nucleic-acid targets, and found that the compounds were also micromolar inhibitors of several unrelated enzymes, including $\beta$-lactamase, chymotrypsin, dihydrofolate reductase and $\beta$-galactosidase. Furthermore, inhibition of various targets was decreased in the presence of bovine serum albumin, a common sign of nonspecific binding. But most intriguingly, the apparent inhibition constants $\left(\mathrm{IC}_{50}\right.$ values $)$ of all the compounds worsened considerably when the concentration of one of the test targets, $\beta$-lactamase, was increased tenfold, in contrast to the behaviour of an established competitive inhibitor.

To account for the extreme sensitivity of the screening hits to the molar ratio of inhibitor to enzyme, the authors considered the hypothesis that the active inhibitor might be an aggregate of many molecules. Dynamic light-scattering experiments (DLS) of aqueous solutions of the hits indicated the presence of particles with apparent diameters ranging from 95 to $400 \mathrm{~nm}$ - much larger than the target enzymes, which are $20 \mathrm{~nm}$ at most in their longest dimension. The presence of aggregates was confirmed by transmission electron microscopy, and also backed up by further experiments on enzyme kinetics.
So, are aggregate-forming nonspecific inhibitors commonplace in corporate screening collections? To investigate this possibility, the authors tested 30 compounds from the Pharmacia Corporation library, biasing the selection of these compounds towards those that register as hits in multiple screens against different targets. Of these 30 , 20 inhibited $\beta$-lactamase and chymotrypsin at micromolar concentrations. As with the previous compounds, inhibition worsened as enzyme concentration was increased, and DLS indicated the presence of large particles.

It thus seems that compounds that inhibit enzymes by forming aggregates could often be artificially raising the hit rates in highthroughput screens for new drug leads. Several important questions remain to be answered, such as how the molecules are arranged in the aggregates, and how the aggregates inhibit the enzymes. Increased understanding should lead to computational or screening methods to rapidly eliminate these phoney hits from future screening programmes.

Peter Kirkpatrick

(2) References and links ORIGINAL RESEARCH PAPER McGovern, S. L. et al. A common mechanism underlying promiscuous inhibitors from virtual and high-throughput screening J. Med. Chem 45, 1712-1722 (2002) 
inactive conformation of the helix.

This new type of antagonism is probably not unique to THC and ER- $\beta$. There are other examples of nuclearreceptor ligands that act as antagonists, even though they might be smaller than the endogenous agonists. Flutamide, a synthetic androgenreceptor antagonist, is similar in size to testosterone, and does not have a bulky side chain to act as an antagonist. Also, progesterone is smaller than aldosterone, but is a high-affinity antagonist of the mineralocorticoid receptor.

This insight into such non-classical antagonism of nuclear receptors highlights a new possible approach to designing antagonists, in which compounds could be tailored to selectively stabilize inactive conformations of certain nuclear receptors, and the active conformations of others. Melanie Brazil

(a) References and links ORIGINAL RESEARCH PAPER Shiau, S. K. etal. Structural characterisation of a subtype-selective ligand reveals a novel mode of estrogen receptor antagonism. Nature Struct. Biol. 9, 359-364 (2002) FURTHER READING Kenakin, T. Efficacy at G-protein-coupled receptors. Nature Rev. Drug Disc. 1, 103-110 (2002) | Christopoulos, A. Allosteric binding sites on cell-surface receptors: novel targets for drug discovery. Nature Rev. Drug Disc. 1, 198-210 (2002)

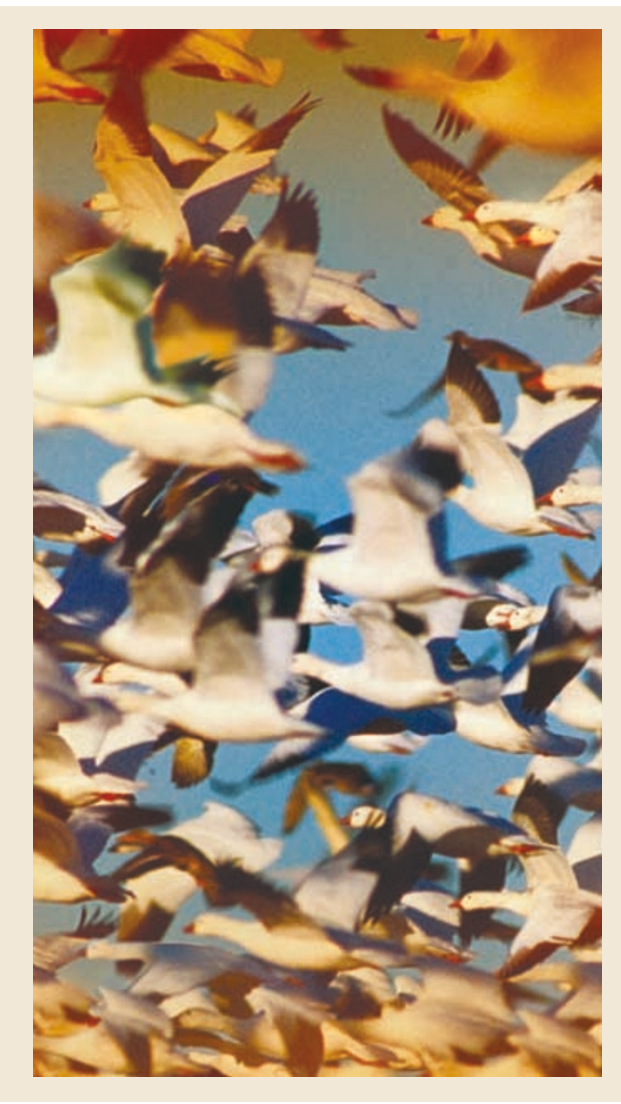

SIGNALLING PATHWAYS

\section{Legless but still the way forward}

The more complex a pathway, the more the output can be fine-tuned by incoming signals.

Unfortunately, this also means that there are more opportunities for things to go wrong, as highlighted by the results of three independent studies led by $\mathrm{He}$, Bienz and Basler.

The Wingless $(\mathrm{Wg})$ — or mammalian WNT pathway transduces signals from the cell surface to the nucleus by preventing the glycogen synthase kinase 3 (GSK3)-mediated phosphorylation of Armadillo/ $\beta$-catenin. When phosphorylated, Armadillo/ $\beta$-catenin gets degraded. However, in the absence of phosphorylation - that is, in response to Wg or WNT - Armadillo/ $\beta$-catenin shuttles to the nucleus to regulate transcription by the T-cell factor, TCF.

He's group studied four key amino-terminal $\beta$-catenin residues - Ser33, Ser37, Thr41 and Ser45 - which, when phosphorylated, target this protein for degradation. The residues conform to a consensus GSK3 phosphorylation site, but does GSK3 phosphorylate them all? The authors showed that a separate 'priming' kinase, casein kinase $\mathrm{I} \alpha(\mathrm{CKI} \alpha)$, was required to phosphorylate Ser45 before GSK3 could phosphorylate the other residues. In its absence, $\beta$-catenin accumulated in the cell. Mutations of axin or the adenomatous polyposis coli (APC) protein, two proteins that allow GSK3 to phosphorylate $\beta$-catenin, are already associated with colon cancer, so perhaps it is not surprising that mutations at Ser33, Ser37, Thr41 or Ser45 are associated with colorectal cancer. The requirement of a priming kinase to allow GSK3 to phosphorylate $\beta$-catenin also has implications for designing therapeutics for other diseases in which GSK3 is implicated, such as type 2 diabetes.

Two more genes - legless and pygopusthat function in the $\mathrm{Wg} / \mathrm{WNT}$ pathway were identified by Basler's group and found to function downstream of Armadillo. Pygopus was also identified by Bienz's group. Legless binds to Armadillo, Pygopus binds to Legless, and Armadillo signalling cannot occur without Legless or Pygopus.
What, then, do these two proteins do? Basler's group showed that Armadillo/ $\beta$ catenin can bind Legless and TCF simultaneously, hinting that Legless and Pygopus might affect Armadillo/ $\beta$-catenin-mediated transcription. As Pygopus enhanced $\beta$-catenin-mediated TCF transcription in tissue-culture cells, this indicates that the principal role of Legless might be to recruit Pygopus to $\beta$-catenin in the nucleus to influence gene transcription.

The Legless protein shows three short regions of homology to the human BCL9 protein, which was subsequently shown to be its functional homologue. The BCL9 gene was originally found juxtaposed to the regulatory elements of an immunoglobulin gene in a cell-line derived from a patient with precursor B-cell acute lymphoblastic leukaemia, in which it was expressed at levels 50-times higher than normal. Although Legless overexpression alone was unable to induce the Wg pathway, higher levels of BCL9 might make cells more sensitive to situations in which the WNT pathway is overactivated, such as the loss of CKI $\alpha$.

Current anticancer drugs aim to disrupt the $\beta$-catenin-TCF complex to inhibit gene transcription, but the authors proposed that targeting the protein-protein interactions between $\beta$-catenin, Legless and Pygopus might be an additional possibility. As proof of principle, mutations that prevent Pygopus binding to Legless inhibited the ability of a mutated form of APC to activate the pathway. Furthermore, drugs aimed to interfere with $\beta$-catenin-TCF binding must be highly specific to avoid disrupting tumoursuppressive $\beta$-catenin-E-cadherin cell-cell adhesions, so targeting the $\beta$-catenin-BCL9 interaction instead might be the way forward.

Katrin Bussell, Nature Reviews Molecular Cell Biology 6) References and links ORIGINAL RESEARCH PAPERS Liu, $C$. et al. Control of $\beta$-catenin phosphorylation/degradation by a dual-kinase mechanism.

Cell 108, 837-847 (2002) | Kramps, T. et al. Wnt/Wingless signaling requires BCL9/Legless-mediated recruitment of Pygopus to the nuclear $\beta$-catenin-TCF complex Cell 109, 47-60 (2002) | Thompson, B. et al. A new nuclear component of the Wnt signalling pathway. Nature Cell Biol. (in the press).

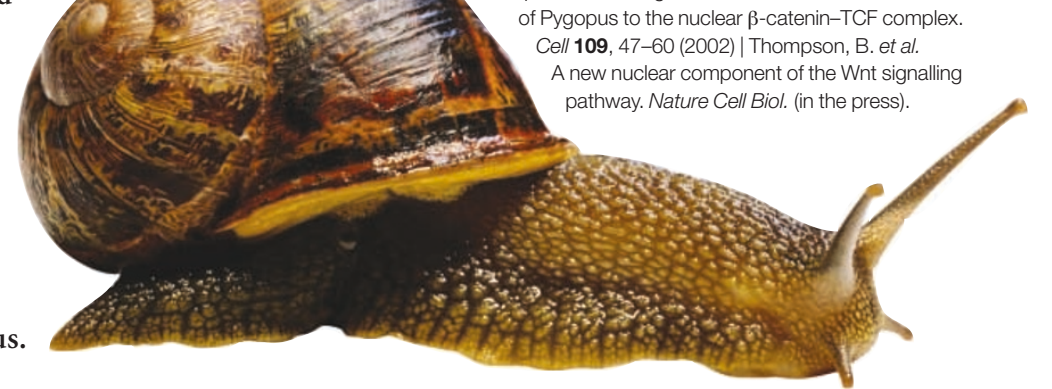

